Research Article

Human and Medical Genetics

\title{
Genetic information improves the prediction of major adverse cardiovascular events in the GENEMACOR population
}

\author{
Maria Isabel Mendonça ${ }^{1}$ (D), Eva Henriques ${ }^{1}$, Sofia Borges ${ }^{1}$, Ana Célia Sousa ${ }^{1}$, Andreia Pereira ${ }^{1}$, \\ Marina Santos ${ }^{1}$, Margarida Temtem ${ }^{1}$, Sónia Freitas ${ }^{1}$, Joel Monteiro ${ }^{1}$, João Adriano Sousa ${ }^{1}$, \\ Ricardo Rodrigues ${ }^{1}$, Graça Guerra ${ }^{1}$ and Roberto Palma dos Reis ${ }^{2}$ \\ ${ }^{1}$ Hospital Central do Funchal, Unidade de Investigação, Serviço de Saúde da Região, SESARAM, EPERAM, \\ Funchal, Portugal. \\ ${ }^{2}$ Universidade Nova de Lisboa, Faculdade de Ciências Médicas, Lisboa, Portugal.
}

\begin{abstract}
The inclusion of a genetic risk score (GRS) can modify the risk prediction of coronary artery disease (CAD), providing an advantage over the use of traditional models. The predictive value of the genetic information on the recurrence of major adverse cardiovascular events (MACE) remains controversial. A total of 33 genetic variants previously associated with CAD were genotyped in 1587 CAD patients from the GENEMACOR study. Of these, 18 variants presented an hazard ratio $>1$, so they were selected to construct a weighted GRS (wGRS). MACE discrimination and reclassification were evaluated by C-Statistic, Net Reclassification Index and Integrated Discrimination Improvement methodologies. After the addition of wGRS to traditional predictors, the C-index increased from 0.566 to 0.572 $(p=0.0003)$. Subsequently, adding wGRS to traditional plus clinical risk factors, this model slightly improved from 0.620 to 0.622 but with statistical significance $(p=0.004)$. NRI showed that $17.9 \%$ of the cohort was better reclassified when the primary model was associated with wGRS. The Kaplan-Meier estimator showed that, at 15-year follow-up, the group with a higher number of risk alleles had a significantly higher MACE occurrence $(p=0.011)$. In CAD patients, wGRS improved MACE risk prediction, discrimination and reclassification over the conventional factors, providing better cost-effective therapeutic strategies.
\end{abstract}

Keywords: Traditional risk factors, genetic risk score, events risk discrimination and reclassification, Net Reclassification Index, secondary prevention of coronary artery disease.

Received: December 07, 2020; Accepted: April 04, 2021.

\section{Introduction}

Coronary artery disease (CAD) is a complex disease with both environmental and hereditary contributions (Hajar, 2017; Said et al., 2019). CAD is the principal cause of morbidity and mortality worldwide. Therefore, it is crucial, for primary prevention, the prompt identification of individuals with an increased risk of developing this condition (Giampaoli et al., 2005). Similarly to other countries, the evaluation of the absolute risk of CAD in the Madeira Archipelago is based on a combination of risk factors that is the basis of modern CAD prevention. It is estimated that the heritability of CAD is around $40 \%-60 \%$, and exhaustive research on genetic predisposition could significantly improve the stratification of CAD risk (Watkins and Farrall, 2006; Khera and Kathiseran, 2017). The genetic variants associated with CAD or its risk factors were previously investigated in the GENEMACOR Study (Pereira et al., 2018a). These were derived from candidate gene studies (Tabor et al., 2002; McCarthy et al., 2004), and GWAS published until 2013, some of them with a known physiopathological mechanism, while others remain under investigation (Hirschhorn and Daly, 2005; Wellcome Trust

Send correspondence to Maria Isabel Mendonça. Hospital Central do Funchal, Unidade de Investigação, Serviço de Saúde da Região Autónoma da Madeira (SESARAM), EPERAM, Avenida Luís de Camões, no 57, 9004-514 Funchal, Portugal E-mail: m.isabel. mendonca@ hotmail.com.
Case Control Consortium, 2007; Hardy and Singleton, 2009; McPherson, 2014). The most relevant genetic variants associated with CAD risk included: $L P A$ (rs3798220), APOE (rs7412/ rs429358), ADIPOQ (rs266729), FTO (rs8050136), MC4R (rs17782313), ACE (rs4340), MTHFR (rs1801133), PON1 (rs854560), TCF21 (rs12190287), PHACTR1 (rs1332844), ZC3HC1 (rs11556924) and CDKN2B-AS1 (rs1333049 and rs4977574) (Pereira et al., 2018b). Incorporating a multilocus genetic risk score (GRS) in commonly used clinical tools has previously improved the prediction of CAD risk (Zhao et al., 2017). Models with traditional risk factors (TRF) performed well in multiple cohorts. However, it was suggested that up to $50 \%$ of individuals with few risk factors and who had been assigned a low risk of developing the disease were, in fact, CAD patients (Futterman and Lemberg, 1998). Current scientific knowledge supports that genetic information can improve these patients' risk stratification. This highpoints the potential role of GRS to complement traditional risk prediction strategies as the GRS is independent of age, and acts long before the onset of clinical risk factors (Benson et al., 2018; Dogan et al., 2018). The patients with established cardiovascular disease (CVD) are prone to suffer from subsequent events, including stroke, myocardial infarction, and death. Prior investigation has verified an association between a multilocus GRS and recurrent cardiovascular events among CAD patients (Jiang et al., 2020). Whether the genetic information adds value to 
conventional and clinical risk factors and influences prognosis remains controversial and inconsistent (Backgr Störk et al., 2006; Patel et al., 2012; Miao et al., 2020).

There is an increasing number of patients living with established CAD due to new pharmacological and interventional therapies (Kandaswamy and Zuo, 2020).

In this work, we investigated whether the addition of genetic variants to conventional risk factors improves the discrimination and reclassification of Major Adverse Cardiovascular Events (MACE) in the context of secondary prevention of CAD in a Portuguese population from GENEMACOR study.

\section{Subjects and Methods}

\section{Subjects}

A total of 1687 consecutive CAD patients were recruited from the Cardiology Department of the Funchal Hospital Centre, which has a regional quality clinical register named "MADEIRA/GESTINTERNMENT", covering more than $90 \%$ of the patients with acute coronary syndrome (ACS) and stable angina (SAP). About 100 patients (5.9\%) were lost to follow-up. The remaining 1587 patients with a diagnosis of CAD were included in the study and allocated to two groups: with and without MACE. They were characterized by coronary angiography showing at least one $\geq 70 \%$ stenosis in any of the main coronary arteries (left anterior descending, left circumflex, and right coronary artery), previous episodes of acute myocardial infarction, coronary revascularization, unstable angina and chronic angina. The exclusion criteria included valvular heart disease, cardiomyopathy, connective tissue disease, severe liver or renal disease and malignant tumours. Patients with a physical disability were also excluded. This prospective cohort is part of the GENEMACOR study, a case-control study taking part in the Madeira Archipelago, which only includes individuals born and living in the Madeira for at least two generations (therefore considered a homogeneous population) (Brehm et al., 2003; Gonçalves et al., 2005).

This study followed the Declaration of Helsinki, and it was approved by the Ethics Committee and Institutional board of Funchal Hospital Centre, under protocol number 50/2012. Written informed consent was attained from all subjects at the time of enrolment.

\section{Cardiovascular outcomes}

The cardiovascular outcomes of this work involved a combination of all-cause vascular morbidity and mortality, including recurrent ACS (myocardial infarction and unstable angina), coronary revascularization (percutaneous or surgical coronary intervention), cardiovascular mortality and readmission due to heart failure or ischemic stroke.

Myocardial infarction (MI), according to the universal definition, is a history of typical ischemic chest pain with increased serum levels of creatinine kinase myocardial band (CK-MB) (greater than 1.5 times) and cardiac troponin above the upper limit of normal (Thygesen et al., 2018). Coronary revascularization was any percutaneous coronary intervention or coronary artery bypass graft procedure performed in the absence of myocardial infarction. Unstable angina (UA) was considered when there is an episode of typical discomfort or pain at rest or during more than 10 minutes or two episodes persisting more than five minutes with negative cardiac biomarkers. Alterations in the electrocardiogram, including 0.5 $\mathrm{mm}$ ST-segment depression or transient ST-segment elevation or $2 \mathrm{~mm}$ T-wave inversion in 2 contiguous leads, may improve this definition specificity of this definition (Braunwald and Morrow, 2013). For cardiovascular mortality, the criteria used is in accordance with the International Classification of Diseases 10th Revision (ICD-10) codes I00-I25, I27, I30-I52, and I60-I72. For ischaemic stroke, ICD-10 codes I63 and I64 were adopted.

In patients with multiple events, only the time of the first event was used for further analyses. Patients were followed-up from $13^{\text {th }}$ March 1999 to $5^{\text {th }}$ September 2019. Two cardiologists independently reviewed all prospective and potential outcomes. Confirmation was achieved on Hospital discharge or deathrelated summary of the events.

\section{Traditional, laboratory and clinical risk factors for MACE}

Covariates of interest include age, gender and the following risk factors:

- $\quad$ Body Mass Index was calculated as weight in kilograms divided by the square of height in meters.

- Smoking status refers to current smokers or subjects having $<5$ years of cessation (Marston et al., 2014).

- CAD patients were classified as diabetic when taking antidiabetic medication or if their fasting glucose was higher than $126 \mathrm{mg} / \mathrm{dL}$ (American Diabetes Association, 2020).

- Dyslipidemia was defined as Low-Density Lipoprotein> $>140$ $\mathrm{mg} / \mathrm{dL}$, High-Density Lipoprotein $<45 \mathrm{mg} / \mathrm{dL}$ for women and $<40 \mathrm{mg} / \mathrm{dL}$ for men, Triglycerides $>150 \mathrm{mg} / \mathrm{dL}$ (Catapano et al., 2016).

- CAD family history is considered if one or more close relatives had early CVD: under 55 for men or before 65 for women (Kolber and Scrimshaw, 2014).

- Physical inactivity was considered a risk factor when subjects practised less than 40 minutes per week of moderate physical activity (Physical Activity Guidelines Advisory Committee Report, 2008).

- Arterial hypertension (ATH) was defined as mean blood pressure of over $140 \mathrm{mmHg}$ (systolic), over $90 \mathrm{mmHg}$ (diastolic), or when blood pressure was controlled, if patients were taking antihypertensive drugs (Mancia et al., 2018).

- The consumption of alcohol in grams per week was quantified and considered significant if it was superior to $60 \mathrm{~g} /$ week in men and $40 \mathrm{~g} /$ week in women. Alcohol abuse was quantified at more than $300 \mathrm{~g} / \mathrm{week}$, which corresponds to exceeding two drinks daily (Rehm et al., 1999).

- All laboratory analyses (fasting glucose, total cholesterol, triglycerides, apolipoprotein B (Apo B), lipoprotein (a), homocysteine, C-reactive protein (hsCRP), fibrinogen, leucocytes and hemoglobin) were carried out in the Clinical Pathology Laboratory of the Central Hospital with quality accreditation, based on the Agencia de Calidad Sanitaria de Andalucía (ACSA) Model (international version). 
- Heart rate was measured by the number of heart beats per minute (bpm) (Spodick et al., 1992).

- Creatinine (cr) clearance was calculated through the Cockcroft Gault' formula (Cockcroft and Gault, 1976).

- Left ventricular ejection fraction (LVEF) was measured during cardiac angiography or by two-dimensional echocardiography. It was based on volume estimation as $\mathrm{LVEF}=[$ (end-diastolic volume - end-systolic volume $)$ $\div$ end-diastolic volume] $\times 100$ (using the apical two- and four-chamber view (Foley et al., 2012).

- Multivessel disease was defined when two or three vessels were affected in contrast to one vessel disease.

\section{Genetic variants selection and genotyping}

Given the large number of genetic variants previously tested in Caucasian populations deriving from multiple studies (candidate genes and GWAS), we only included genes associated with CAD and already investigated in the GENEMACOR study. In total, we considered 33 genetic variants with a Minor Allele Frequency (MAF) > 2\% (Kim et al. 2011), which were distributed by five major physiopathological axes (Table S1), according to their most consensual action pathway in coronary atherosclerosis: six single nucleotide polymorphisms (SNPs) in the lipid metabolism axis corresponding to PSRC1 (rs599839), PCSK9 (rs2114580), KIF6 (rs20455), LPA (rs3798220), ZPR1 (rs964184) and APOE (rs7412/rs429358); nine SNPs in Diabetes/Obesity and Insulin Resistance axis, namely $A D I P O Q$ (rs266729), IGF2BP2 (rs4402960), $P P A R G$ (rs1801282), SLC30A8 (rs1326634), TCF7L2 (rs7903146), TAS2R50 (rs1376251), FTO (rs8050136), MC4R (rs17782313) and HNF4A (rs1884613); three SNPs in the Hypertension (Renin-Angiotensin-Aldosterone) axis, namely $A G T$ (rs699), $A G T 1 R$ (rs5186) and $A C E$ (rs4340); six SNPs were associated with the pro-oxidative state (Oxidation), namely MTHFR (rs1801131 and rs1801133), MTHFDIL (rs6922269), PON1 (rs705379, rs662 and rs854560). Finally, nine SNPs whose pathophysiological mechanism is not fully understood and might be involved in cell cycle, genetic transcription, smooth muscle cells differentiation and proliferation or acting as cotransport binders (Cellular): MIA3 (rs17465637), GJA4 (rs618675), TCF21 (rs12190287), PHACTR1 (rs1332844), ZC3HC1 (rs11556924), CDKN2B-AS1 (rs1333049 and rs4977574), SMAD3 (rs17228212) and ADAMTS7 (rs3825807) (Assimes and Roberts, 2016) (Table S1).

A TaqMan allelic discrimination assay for genotyping was performed using labelled probes and primers preestablished by the supplier (TaqMan SNP Genotyping Assays, Applied Biosystems). All reactions were done on an Applied Biosystems 7300 Real-Time PCR System and genotypes were determined using the 7300 System SDS Software (Applied Biosystems, Foster City, USA) without any prior knowledge of the individual's clinical data. Quality check of genotyping techniques was maintained by the inclusion of one nontemplate control (NTC) in each plate of 96 wells. All SNPs TaqMan assays had blind duplicates accounting for $20 \%$ of all samples. Some SNP genotypes were randomly confirmed by conventional direct DNA sequencing, as $10-15 \%$ of all samples were re-amplified for sequencing.

\section{Statistical analysis}

\section{Descriptive and comparative analysis}

Continuous variables were defined as means $( \pm \mathrm{SD})$ or medians $(\mathrm{Q} 1-\mathrm{Q} 3)$, as appropriate, and categorical variables were determined as frequencies and proportions. We used the t-Student test (or Mann-Whitney) to compare continuous data and $\chi^{2}$ tests to compare categorical variables.

\section{Construction of $w G R S$}

For constructing an additive wGRS, 33 SNPs (Table S1) were surveyed for association with MACE in a Cox proportional hazard model. Those SNPs with a Hazard Ratio (HR) $>1$ were selected for the additive GRS. Subsequently, we counted the number of risk alleles for each of these SNPs, and the genotypes were coded as " 0 ", " 1 " and " 2 " for wild homozygous, heterozygous, and mutated homozygous, respectively. The additive weighted GRS (wGRS) was achieved by summing the product between the HR for each SNP and the number of risk alleles $(0,1,2)$.

\section{WGRS discriminative capacity to predict MACE}

To estimate the wGRS discriminative capacity in MACE prediction, two analysis were performed: firstly, using TRFs as the baseline model, wGRS and clinical risk factors were sequentially added and compared; secondly, using TRFs + clinical risk factors as the baseline, wGRS was included and then compared. Harrell's C-statistical approach tested the area under the Receiving Operating curve (ROC) of the models with and without wGRS and its statistical significance. C-statistic refers to the probability that predicting the outcome is better than chance comparing Cox regression models. Calibration was verified by Hosmer and Lemeshow goodness-of-fit test.

\section{MACE reclassification}

Net Reclassification Improvement (NRI) was calculated according to the continuous method. The number of individuals reclassified into higher and lower risk was applied to the two models with and without wGRS. NRI was designated as the percentage of subjects whose risk is changed (upwards or downwards) when adding the new marker (wGRS). Integrated Discrimination Improvement (IDI) can be defined as an increment of the difference between the means of predicted probabilities of two models: with and without the added marker (wGRS) (Steyerberg et al., 2010; Pencina et al., 2012).

\section{Cumulative hazard rates according to $w G R S$}

Event-free survival time was defined as the interval between the admission date to the study and the first event of interest. Patients who had not experienced MACE by the time of the last follow-up were censored. Unadjusted survival and its cumulative hazard curves for each of the outcomes were created by Kaplan-Meier estimator. Breslow test was performed to evaluate the differences between the high (higher than the median) and low (lower than the median) risk MACE groups.

The statistics methods used were those of the Statistical Package for the Social Sciences software version 25.0 (IBM, Armonk, NY, USA), MedCalc version 13.3.3.0 and R (version 3.2.0). All $P$-values were two-sided, statistically significant for $\mathrm{p}<0.05$. 


\section{Results}

\section{Study population characteristics: patients with and without MACE}

A total of 1587 coronary patients were allocated to two groups: 567 who experienced MACE (35.7\%) and 1020 $(64.3 \%)$ without MACE. MACE included non-fatal myocardial infarction, unstable angina, revascularization (percutaneous coronary intervention PCI and coronary artery bypass grafting $\mathrm{CABG}$ ), heart failure and ischemic stroke, were documented during the interquartile range (IQR) of 5.7 ( $\min 0.2-\max$ 20.5) years of follow-up. Of these patients with MACE, 212 $(37.4 \%)$ resulted in cardiovascular mortality.

The demographic, traditional, laboratory and clinical features of the study population are summarised in Table 1.

Patients who experienced MACE were older than those without, but the percentage of men was similar in both groups. These patients had a more aggressive atherogenic profile when compared to patients who did not suffer MACE. They had a higher prevalence of the some of the most critical risk factors for CAD: diabetes (41.6\% vs 30.7\%), dyslipidemia $(91.7 \%$ vs $87.8 \%$ ), physical inactivity $(71.8 \%$ vs $58.9 \%)$, arterial hypertension $(76.0 \%$ vs $68.4 \%)$ and alcohol intake $>300 \mathrm{gr} /$ week $(21.3 \%$ vs $14.3 \%)$ (all $\mathrm{p}<0.05)$. Risk factors, such as fasting glucose, triglycerides, apo B, lipoprotein (a) $>30 \mathrm{mg} /$ $\mathrm{dL}, \mathrm{CRP}>3 \mathrm{mg} / \mathrm{L}$ and fibrinogen were presented with higher values in the MACE cohort $(\mathrm{p}<0.05)$. Inversely, smoking status and HDL cholesterol were more elevated in the nonMACE group. The variables associated with clinical risk, including heart rate, creatinine clearance $<60 \mathrm{ml} / \mathrm{min}$, ejection fraction $<55$ and multivessel disease, were significantly higher in the cohort of patients with MACE (all p<0.0001) (Table 1).

\section{Genetic risk for MACE in CAD patients}

For the construction of an additive wGRS, 33 SNPs associated with CAD risk from the GENEMACOR study were analysed (Table S1). Although none of these reached statistical significance for MACE risk using a Cox multivariate analysis, 18 SNPs were selected for wGRS since they presented a MACE risk $(\mathrm{HR})>1$ (Table 2). These were: LPA (rs3798220), ZPR1 (rs964184), IGF2BP2 (rs4402960), TCF7L2 (rs7903146), TAS2R50(rs1376251), FTO(rs8050136), MC4R(rs17782313), AGT (rs699), AGT1R (rs5186), MTHFR (rs1801131), PON1 (rs705379), MIA3 (rs17465637), GJA4 (rs618675), TCF21 (rs12190287), PHACTR1 (rs1332844), ZC3HCl (rs11556924), $C D K N 2 B-A S 1$ (rs1333049 and rs4977574) (Table 2).

WGRS ranged from 25.1 to 42.1 , with a mean of $33.7 \pm$ 2.8 for patients with MACE and 33.2 \pm 2.9 for those without MACE $(\mathrm{p}<0.0001)$ (Table 1$)$. The curve distribution of wGRS in patients with events (risk allele distribution) is moved to the right of the picture compared to patients' curve without events (Figure 1).

To evaluate the independent association of the wGRS with the risk of MACE, two groups above and below the median were considered, and three Cox proportional-hazards models were derived. The first was adjusted to age and gender, and the second to the traditional co-variables (gender, age, BMI, smoking, diabetes, dyslipidemia, hypertension and physical inactivity). The third model was adjusted to the same conventional factors plus other clinical co-variables (CRP, ejection fraction, multivessel disease and creatinine clearance). WGRS was significantly associated with MACE risk: the first one with an HR of 1.373, the second with an HR of 1.371 and the third with an HR of 1.293 (Figure 2).

\section{Comparison of the individual predictive capacity of the different risk factors (traditional, clinical and genetic) to the risk of MACE}

We evaluated the individual predictive ability of MACE risk for each traditional, laboratory, clinical and genetic risk factors, using proportional-Hazard Cox multivariate analysis and $\mathrm{C}$-statistic methodology.

After Cox multivariate analysis, all risk factors, except gender, body mass index, dyslipidemia, and smoking, were independently correlated with the occurrence of MACE. The individual HR is exposed in Table 3. It is noted that the wGRS exhibits a more significant correlation to the risk of MACE than dyslipidemia, diabetes, arterial hypertension and decreased renal function. It is only surpassed by $\mathrm{CRP}>3 \mathrm{mg} / \mathrm{L}$, physical inactivity, low ejection fraction and multivessel disease (Table 3).

Using ROC curve for events discrimination (C-statistic), the results are similar to the previous analysis, excepting diabetes, which appears along with the investigated variables yielding a slight improvement for predicting events compared to wGRS (Figure 3).

\section{Prediction and discrimination of total MACE: models with and without wGRS}

For total MACE discrimination, we used a C-Statistic methodology. The model was developed for the composite outcomes of an ACS (MI and UA), revascularization, heart failure, ischemic stroke and death. It was adjusted for all studied traditional and clinical risk factors. When the wGRS was incorporated in the baseline model (with conventional risk factors only), the ROC curve for events discrimination (C-Statistic) increased from 0.566 (95\% CI: 0.539-0.593) to $0.572(95 \% \mathrm{CI}: 0.545-0.599)(\mathrm{p}=0.0003)$. The prognostic $\mathrm{C}$-index was $0.6 \%$. However, when wGRS plus clinical factors were incorporated in the baseline model, it increased from 0.566 to $0.622(95 \%$ CI: $0.595-0.649)(\mathrm{p}<0.0001)$ with a prognostic C-index of $5.6 \%$ (Table 4 ).

Subsequently, adding wGRS to traditional plus clinical risk factors, this model slightly improved from 0.620 to 0.622 but with statistical significance $(\mathrm{p}=0.004)$ (Table 4$)$.

\section{Discrimination and Reclassification of MACE with NRI and IDI methodology}

We used the new statistical metrics NRI and IDI to evaluate the progression of discrimination when the model with traditional and clinical information is added to wGRS. The continuous based NRI added to wGRS improved the nongenetic model discrimination, which was confirmed by the IDI.

Model calibration was tested by goodness-of-fit with the Hosmer and Lemeshow test and was suitable for the model predicting MACE $(\mathrm{p}=0.461)$.

Using NRI, the net proportion of events (NRIe) that assigned a higher risk was $25.9 \%$ (better reclassified), and the net ratio of non-events (NRIne) was $-8.0 \%$. This result 
reflects an increased percentage of non-events going to a higher category (worst reclassified), resulting in a total NRI of $17.9 \%$ of patients being better reclassified when genetic information was included (Table 4). IDI was $0.7 \%$ showing a difference in predicted probabilities between patients with and without events.

\section{Cumulative hazard rates according to wGRS}

Remarkably, the Kaplan-Meier curve analysis at 15-years follow-up showed the group with a higher number of risk alleles (higher than the median) had a significantly higher occurrence of MACE and worst lifetime (15.9\%) when compared to the lower than the median $(27.4 \%)$ with a fewer number of risk alleles (Figure 4A). The comparison of the two curves was statistically significant with the Breslow test $(p=0.011)$. However, there were no substantial differences between the probability of survival event-free lifetime from the two groups after 18-years of follow-up, and both presented approximately $10 \%$ of event-free chance. The cumulative hazard curves showed a higher risk in the group above the median of wGRS ( $\mathrm{p}=0.011)$ through the years, being similarly at 18-years, approximately (Figure 4B).

Table 1 - Overall characteristics of the study population.

\begin{tabular}{|c|c|c|c|c|}
\hline Overall characteristics & $\begin{array}{c}\text { Overall } \\
(n=1587)\end{array}$ & $\begin{array}{l}\text { MACE } \\
(n=567)\end{array}$ & $\begin{array}{c}\text { No-MACE } \\
(n=1020)\end{array}$ & P-value** \\
\hline \multicolumn{5}{|l|}{ Demographic } \\
\hline Age, years & $53.3 \pm 7.9$ & $54.0 \pm 7.6$ & $52.9 \pm 8.1$ & 0.006 \\
\hline Male sex, n (\%) & $1246(78.5)$ & $445(78.5)$ & $801(78.5)$ & 0.983 \\
\hline BMI, kg/m² & $28.6 \pm 4.3$ & $28.7 \pm 4.2$ & $28.6 \pm 4.4$ & 0.826 \\
\hline \multicolumn{5}{|l|}{ Traditional risk factors } \\
\hline Smoking status, n (\%) & $753(47.4)$ & $241(42.5)$ & $512(50.2)$ & 0.003 \\
\hline Diabetes, n (\%) & $549(34.6)$ & $236(41.6)$ & $313(30.7)$ & $<0.0001$ \\
\hline Dyslipidemia, n (\%) & $1416(89.2)$ & $520(91.7)$ & $896(87.8)$ & 0.017 \\
\hline CAD family history, $\mathrm{n}(\%)$ & $381(24.0)$ & $144(25.4)$ & $237(23.2)$ & 0.334 \\
\hline Physical inactivity, n (\%) & $1008(63.5)$ & $407(71.8)$ & $601(58.9)$ & $<0.0001$ \\
\hline Hypertension, n (\%) & $1129(71.1)$ & $431(76.0)$ & $698(68.4)$ & 0.001 \\
\hline Alcohol>300gr/week, n (\%) & $267(16.8)$ & $121(21.3)$ & $146(14.3)$ & $<0.0001$ \\
\hline SBP, mmHg & $137.6 \pm 20.6$ & $137.8 \pm 20.7$ & $137.5 \pm 20.5$ & 0.763 \\
\hline DBP, $\mathrm{mmHg}$ & $82.4 \pm 11.8$ & $81.6 \pm 11.4$ & $82.9 \pm 12.0$ & 0.043 \\
\hline \multicolumn{5}{|l|}{ Laboratory Risk Factors } \\
\hline Fasting glucose, $\mathrm{mg} / \mathrm{dL}$ & $106.0(9.0-130.0)$ & $110.0(98.0-138.0)$ & $104.0(96.0-125.0)$ & $<0.0001$ \\
\hline Total cholesterol, mg/dL & $180.0(155.0-211.0)$ & $184.0(155.0-216.0)$ & $179.0(154.5-207.5)$ & 0.060 \\
\hline $\mathrm{LDL}, \mathrm{mg} / \mathrm{dL}$ & $105.1(83.2-127.4)$ & $107.0(83.6-129.4)$ & $104.7(82.7-126.3)$ & 0.212 \\
\hline $\mathrm{HDL}, \mathrm{mg} / \mathrm{dL}$ & $42.0(35.3-49.0)$ & $40.0(34.5-48.0)$ & $42.0(36.0-49.0)$ & 0.002 \\
\hline Triglycerides, mg/dL & $140.0(103.0-207.0)$ & $146.0(108.0-214.0)$ & $136.0(101.5-202.5)$ & 0.031 \\
\hline Apolipoprotein B, mg/dL & $93.4(77.0-111.8)$ & $97.4(79.8-116.6)$ & $93.4(75.3-109.4)$ & $<0.0001$ \\
\hline Lipoprotein (a)>30mg/dL, n (\%) & $620(39.1)$ & $255(45.0)$ & $365(35.8)$ & $<0.0001$ \\
\hline Homocysteine >10mg/dL, n (\%) & $1217(76.7)$ & $445(78.5)$ & $772(75.7)$ & 0.207 \\
\hline $\mathrm{CRP}>3 \mathrm{mg} / \mathrm{L}, \mathrm{n}(\%)$ & $616(38.8)$ & $293(51.7)$ & $323(31.7)$ & $<0.0001$ \\
\hline Fibrinogen, mg/dL & $388.0(340.4-446.0)$ & $397.0(342.0-462.0)$ & $383.0(339.5-440.0)$ & 0.009 \\
\hline Leucocytes, mg/dL & $7.1(6.1-8.4)$ & $7.2(6.1-8.5)$ & $7.1(6.0-8.2)$ & 0.123 \\
\hline Hemoglobin, mg/dL & $14.6(13.8-15.3)$ & $14.6(13.7-15.2)$ & $14.6(13.8-15.4)$ & 0.142 \\
\hline \multicolumn{5}{|l|}{ Clinical risk factors } \\
\hline Heart rate, beat/min. & $68.6 \pm 12.6$ & $70.3 \pm 13.6$ & $67.7 \pm 11.9$ & $<0.0001$ \\
\hline Cr Clearance $*<60 \mathrm{ml} / \mathrm{min}, \mathrm{n}(\%)$ & $123(7.8)$ & $65(11.5)$ & $58(5.7)$ & $<0.0001$ \\
\hline Ejection fraction< $55, \mathrm{n}(\%)$ & $450(28.4)$ & $226(39.9)$ & $224(22.0)$ & $<0.0001$ \\
\hline Multivessel disease, n (\%) & $788(49.7)$ & $361(63.7)$ & 427 (41.9) & $<0.0001$ \\
\hline \multicolumn{5}{|l|}{ Genetic information } \\
\hline wGRS & $33.4 \pm 2.9$ & $33.7 \pm 2.8$ & $33.2 \pm 2.9$ & $<0.0001$ \\
\hline
\end{tabular}

MACE - Major Adverse Cardiovascular Events; BMI - Body mass index; CAD - Coronary artery disease; SBP - Systolic blood pressure; DBP - Diastolic blood pressure; LDL - Low-density lipoprotein; HDL - High-density lipoprotein; CRP - C reactive protein; Cr Clearance - Creatinine Clearance; *CockroftGault $<60 \mathrm{ml} / \mathrm{min}$.; wGRS - Weighted genetic risk score; Continuous variables presented as mean $\pm \mathrm{SD}$ or median (IQR(Q1 - Q3)); **P-values from the Chi-square test for categorical variables and t Student or Mann-Whitney tests for continuous variables, as appropriate; Statistically significant for $\mathrm{p}<0.05$. 
Table 2 - Genetic variants associated with MACE occurrence.

\begin{tabular}{|c|c|c|}
\hline Genetic Variants & Hazard ratio $(95 \% \mathrm{CI})$ & P-value \\
\hline LPA rs3798220 & $1.095(0.909-1.320)$ & 0.340 \\
\hline ZPR1 rs 964184 & $1.021(0.875-1.191)$ & 0.795 \\
\hline$I G F 2 B P 2$ rs 4402960 & $1.130(0.992-1.287)$ & 0.065 \\
\hline TCF7L2 rs 7903146 & $1.018(0.896-1.156)$ & 0.783 \\
\hline TAS2R50 rs 1376251 & $1.051(0.878-1.258)$ & 0.587 \\
\hline FTO rs 8050136 & $1.074(0.951-1.212)$ & 0.251 \\
\hline$M C 4 R$ rs 17782313 & $1.058(0.924-1.211)$ & 0.413 \\
\hline$A G T r s 699$ & $1.051(0.934-1.184)$ & 0.408 \\
\hline$A G T 1 R$ rs 5186 & $1.085(0.947-1.243)$ & 0.238 \\
\hline MTHFR rs 1801131 & $1.045(0.902-1.211)$ & 0.558 \\
\hline PON1 rs 705379 & $1.046(0.910-1.203)$ & 0.524 \\
\hline MIA3 rs 17465637 & $1.096(0.959-1.253)$ & 0.179 \\
\hline GJA4 rs 618675 & $1.018(0.883-1.173)$ & 0.806 \\
\hline TCF 21 rs 12190287 & $1.130(0.998-1.287)$ & 0.050 \\
\hline PHACTR1 rs 1332844 & $1.027(0.912-1.158)$ & 0.657 \\
\hline ZC3HC1 rs 11556924 & $1.068(0.939-1.215)$ & 0.317 \\
\hline$C D K N 2 B-A S 1$ rs 1333049 & $1.031(0.784-1.357)$ & 0.826 \\
\hline$C D K N 2 B-A S 1$ rs 4977574 & $1.041(0.789-1.374)$ & 0.776 \\
\hline
\end{tabular}

MACE - Major Adverse Cardiovascular Events; CI - Confidence interval;Statistically significant for p $<0.05$. WGRS calculated by the formula: $\Sigma($ HR $\times$ Number of risk alleles).

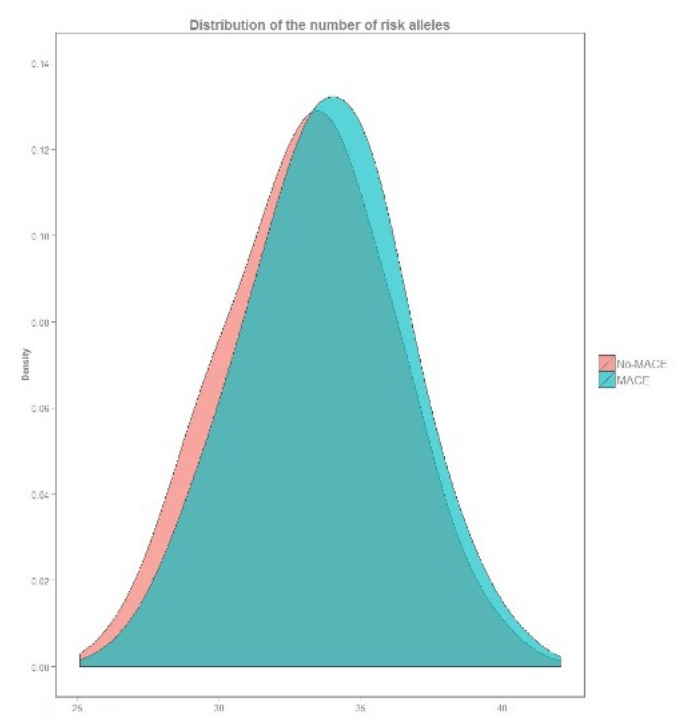

Figure 1 - Distribution of the number of risk alleles in CAD patients with and without MACE.

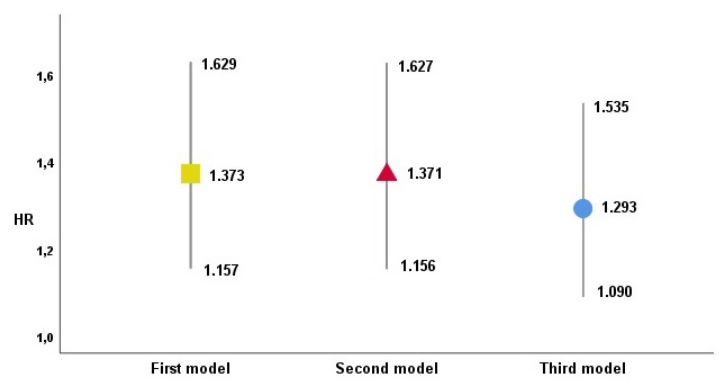

Figure 2 - Risk of MACE occurrence with different wGRS adjustments.

wGRS Hazard ratios (HR) from Cox regression analysis for prediction of MACE on 3 different models: $1^{\text {st }}$ adjusted for age and gender, $2^{\text {nd }}$ including TRFs and $3^{\text {rd }}$ including TRFs and clinical variables. Statistically significant for each model $(p<0.0001 ; p<0.0001$ and $p=0.003$, respectively). 
Table 3 - Individual MACE risk (HR) of all studied variables.

\begin{tabular}{|c|c|c|}
\hline Overall characteristics & $\mathrm{HR}(95 \% \mathrm{CI})$ & P-value** \\
\hline \multicolumn{3}{|l|}{ Demographic } \\
\hline Age, years & $1.012(1.002-1.023)$ & 0.023 \\
\hline Male sex, n (\%) & $0.859(0.703-1.050)$ & 0.139 \\
\hline BMI, $\mathrm{kg} / \mathrm{m}^{2}$ & $1.009(0.990-1.028)$ & 0.365 \\
\hline \multicolumn{3}{|l|}{ Traditional risk factors } \\
\hline Smoking status, n (\%) & $0.931(0.788-1.101)$ & 0.405 \\
\hline Diabetes, n (\%) & $1.300(1.099-1.537)$ & 0.002 \\
\hline Dyslipidemia, n (\%) & $1.134(0.841-1.529)$ & 0.410 \\
\hline CAD family history, n (\%) & $0.889(0.735-1.076)$ & 0.226 \\
\hline Physical inactivity, n (\%) & $1.453(1.210-1.744)$ & $<0.0001$ \\
\hline Hypertension, n (\%) & $1.313(1.083-1.592)$ & 0.006 \\
\hline Alcohol>300gr/week, n (\%) & $1.374(1.123-1.681)$ & 0.002 \\
\hline $\mathrm{SBP}, \mathrm{mmHg}$ & $0.998(0.994-1.002)$ & 0.348 \\
\hline DBP, mmHg & $0.995(0.988-1.002)$ & 0.176 \\
\hline \multicolumn{3}{|l|}{ Laboratory Risk Factors } \\
\hline Fasting glucose, mg/dL & $1.002(1.001-1.004)$ & 0.002 \\
\hline Total cholesterol, mg/dL & $1.000(0.998-1.002)$ & 0.911 \\
\hline $\mathrm{LDL}, \mathrm{mg} / \mathrm{dL}$ & $0.999(0.997-1.001)$ & 0.323 \\
\hline $\mathrm{HDL}, \mathrm{mg} / \mathrm{dL}$ & $0.989(0.982-0.997)$ & 0.007 \\
\hline Triglycerides, mg/dL & $1.001(1.000-1.001)$ & 0.042 \\
\hline Apolipoprotein B, mg/dL & $1.001(0.998-1.003)$ & 0.652 \\
\hline Lipoprotein (a)>30mg/dL, n (\%) & $1.163(0.985-1.374)$ & 0.074 \\
\hline Homocysteine>10mg/dL, n (\%) & $1.001(0.819-1.225)$ & 0.989 \\
\hline $\mathrm{CRP}>3 \mathrm{mg} / \mathrm{L}, \mathrm{n}(\%)$ & $1.408(1.193-1.661)$ & $<0.0001$ \\
\hline Fibrinogen, mg/dL & $1.002(1.001-1.003)$ & $<0.0001$ \\
\hline Leucocytes, mg/dL & $1.049(1.010-1.089)$ & 0.014 \\
\hline Hemoglobin, mg/dL & $0.926(0.870-0.987)$ & 0.017 \\
\hline \multicolumn{3}{|l|}{ Clinical risk factors } \\
\hline Heart rate, beat $/ \mathrm{min}$ & $1.011(1.004-1.017)$ & 0.001 \\
\hline Cr Clearance* $<60 \mathrm{ml} / \mathrm{min}, \mathrm{n}(\%)$ & $1.349(1.040-1.749)$ & 0.024 \\
\hline Ejection fraction $<55, \mathrm{n}(\%)$ & $1.650(1.393-1.955)$ & $<0.0001$ \\
\hline Multivessel disease, n (\%) & $1.908(1.608-2.265)$ & $<0.0001$ \\
\hline \multicolumn{3}{|l|}{ Genetic information } \\
\hline wGRS & $1.371(1.155-1.627)$ & $<0.0001$ \\
\hline
\end{tabular}

MACE - Major adverse coronary events; HR - Hazard Ratio; CI - Confidence Interval; BMI - Body mass index; CAD - Coronary artery disease; SBP - Systolic blood pressure; DBP - Diastolic blood pressure; LDL - Low density lipoprotein; HDL - High density lipoprotein; CRP - C reactive protein; Cr Clearance - Creatinine Clearance; *Cockroft-Gault $<60 \mathrm{ml} / \mathrm{min}$; wGRS -Weighted genetic risk score; ${ }^{* *}$ Cox regression analysis entering each variable individually; Statistically significant for $\mathrm{p}<0.05$.

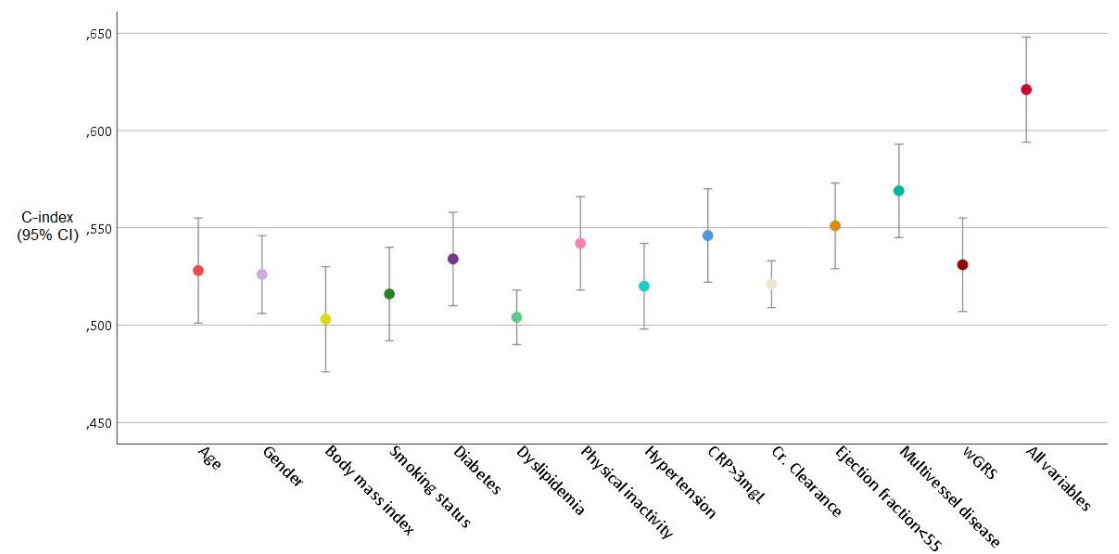

Figure 3 - Individual predictive capacity of the investigated variables to MACE risk by C-index methodology. 
Table 4 - MACE discrimination using Harrel's C-statistic, NRI and IDI methodology.

\begin{tabular}{|c|c|c|c|}
\hline Models & & C-index $(95 \% \mathrm{CI})$ & P-value \\
\hline TRFs & & $0.566(0.539-0.593)$ & \\
\hline TRFs + wGRS & & $0.572(0.545-0.599)$ & $0.0003^{(a)}$ \\
\hline TRFs + clinical + wGRS & & $0.622(0.595-0.649)$ & $<0.0001^{(\text {b) }}$ \\
\hline TRFs + clinical & & $0.620(0.593-0.647)$ & \\
\hline TRFs + clinical + wGRS & & $0.622(0.595-0.649)$ & $0.004^{(\mathrm{c})}$ \\
\hline \multirow{7}{*}{ TRFs + clinical + wGRS } & \multicolumn{3}{|c|}{ NRI } \\
\hline & Total & MACE & \multirow{2}{*}{$\begin{array}{c}\text { Non-MACE } \\
-8.0 \% \\
(-14.2 \%-(-1.9 \%))\end{array}$} \\
\hline & $\begin{array}{c}17.9 \% \\
(7.9 \%-27.9 \%)\end{array}$ & $\begin{array}{c}25.9 \% \\
(18.0 \%-33.9 \%)\end{array}$ & \\
\hline & $p=0.0005$ & $\mathrm{p}<0.0001$ & $p=0.010$ \\
\hline & \multicolumn{3}{|c|}{ IDI } \\
\hline & $\begin{array}{c}0.7 \% \\
(0.2 \%-1.0 \%)\end{array}$ & \multicolumn{2}{|l|}{-} \\
\hline & $\mathrm{p}=\mathbf{0 . 0 0 2}$ & - & - \\
\hline
\end{tabular}

MACE - Major adverse coronary event; NRI - Net reclassification index; IDI - Integrated discrimination index; TRFs - Traditional Risk Factors; wGRS - Weighted genetic risk score; Clinical - Clinical risk factors; CI - Confidence interval. (a) Comparison among TRFs+wGRS vs TRFs models; (b) Comparison between TRFs+clinical+wGRS vs TRFs models; (c) Comparison between TRFs+clinical+wGRS vs TRFs+clinical models. Statistically significant for $\mathrm{p}<0.05$.
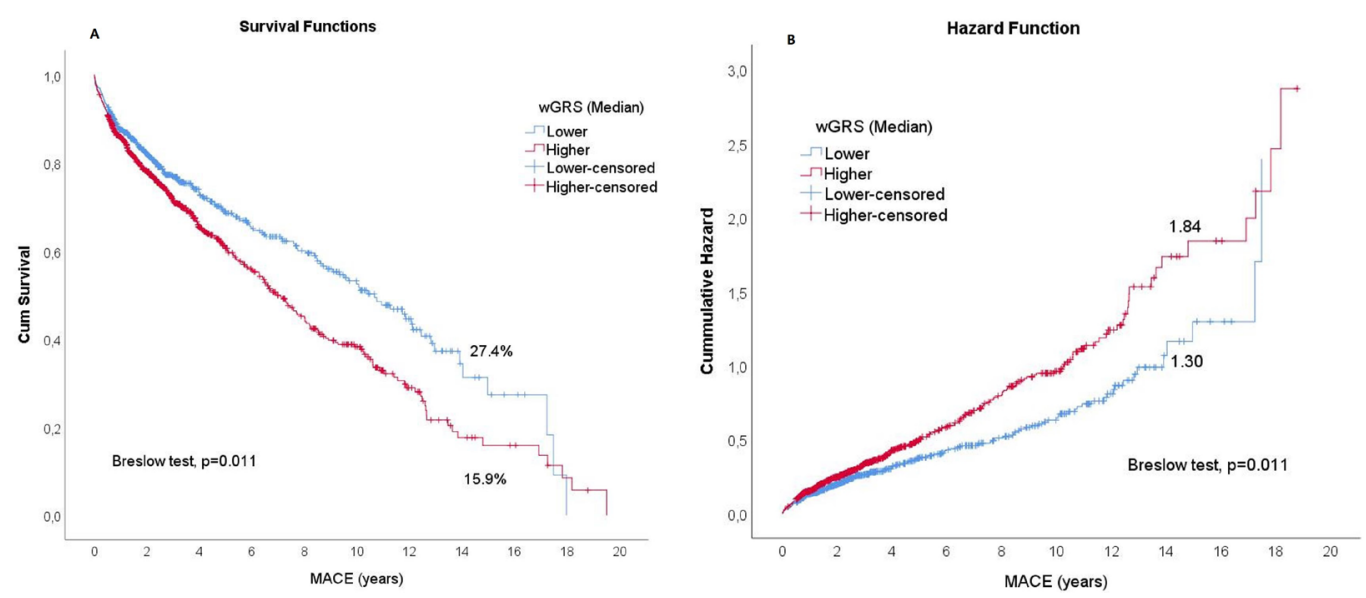

Figure 4 - Survival analysis for patients with and without MACE, comparing wGRS above and below the median.

(A) Cumulative survival curves: Lower- and higher-censored data are the individuals who, at the time of follow-up, didn't develop any type of MACE in each wGRS categories (Lower and higher than median).

(B) Cumulative hazard curves: Lower- and higher-censored data are the individuals who, at the time of follow-up, didn't develop any type of MACE in each wGRS categories (Lower and higher than median).

\section{Discussion}

In CAD patients, the recurrence of myocardial infarction or other cardiovascular events increases morbidity and mortality. Tools have been developed to predict recurrent or possibly lethal ischemic events, including traditional and clinical risk factors. Unfortunately, risk prediction based on these tools alone is not robust enough, and it was thought that genetic information could improve this ability. Although a prior investigation has shown the predictive value of a multilocus genetic risk score to recurrent cardiovascular events in CAD patients, this subject remains controversial and inconsistent (Labos et al., 2015; Smit et al., 2015). As far as we know, the present study is the first in Portugal to investigate the cumulative capacity of genetic variations to predict the risk of MACE occurring over a long period of follow-up in patients with known CAD. Long-term cohorts provide more reliable estimates of survival rates in populations and offer an excellent opportunity to estimate the association between genetic information and specific MACE occurrence in coronary disease.

Given the high burden of recurrent ischemic events and in light of the controversies surrounding this issue, we decided to investigate whether the incorporation of genetic risk factors in a model with traditional and clinical risk factors improved the discriminatory performance of the MACE risk forecast and improved the risk of the patient with CAD installed. We developed an 18-wGRS, based on 33 SNPs previously associated with $\mathrm{CAD}$, being correlated with the risk of adverse cardiovascular events.

This score, tested in three models, was significantly and independently associated with MACE risk. 
We have used different methodologies to evaluate our wGRS to discriminate the risk of MACE when incorporated in the traditional risk prediction tools. It was shown that although genetic factors have a significant role in the risk of MACE occurrence, the clinical variables have the highest value in this prediction. C-statistic was not as accurate as the NRI method. A prior simulation study showed the method of testing differences used in C-index is not recommended in situations where the added value of the new predictor is not very significant. Massive associations are required to increase the predictive performance significantly (Delong et al., 1988; Demler et al., 2012). To overcome these drawbacks we applied the indexes (NRI and IDI) proposed by Pencina et al. (2012), which more accurately quantify the incremental value of the predictors. The incorporation of genetic information into traditional models, evaluated by NRI and IDI, improved the risk reclassification, which became more discriminatory, accurate and clinically valuable. Specifically, when the genetic information was included in the traditional models (classical plus clinical), $17.9 \%$ of our population were better reclassified. These results highlight the importance of considering the addition of a new marker to an established model. NRI methodology, in our opinion, is a more accurate and clinically helpful methodology for quantifying and classifying these patients into different risk groups. Besides, reclassification to a higher risk group allows medical decision-makers to treat and monitor them more aggressively. Genetic information inclusion has more potential to identify patients at high-risk than conventional risk evaluation alone and improve clinical practice (Moorthie et al., 2019). However, the minimal degree of improvement assigned by incorporating the wGRS into established risk tools needed for clinical utility is unknown, and there are still questions about the definitive clinical usefulness of GRS. Additional investigation in high-risk populations incorporating new data from genomics and gene expression studies in developing new genetic risk scores may be helpful. Likewise, new statistical approaches based on data mining and artificial intelligence transform future prediction techniques (Chan et al., 2018).

The cumulative rates of risk events, according to the wGRS, remain controversial. These contradictions can be motivated by different approaches in the evaluation of the results. When the analysis focuses on the initial period, we can get quite different results from those found at the end of an extensive follow-up. In the present study, cumulative risk rate analysis showed that the group with the highest risk alleles was associated with a significantly higher incidence of cardiovascular events and worse event-free lifetime. However, sooner or later, if they live enough, all patients start having events. In the early stages of life, genetic susceptibility to CAD (heritability) has a significant impact, but this propensity "loses traction" over time, as it competes with lifestyle and environmental factors, medications, and other comorbidities. Some initially lower-risk individuals, over an extended followup, get older, and degenerative CAD mechanisms go forward exhausting all therapeutic options for chronic CVD (statins, antihypertensives, and revascularization) starting to behave in a similar way to higher-risk patients. So, at the end of a long follow-up the two survival curves overlap.
Our work's GRS emphasizes the concept that genetic information can be more useful in early life, stratifying individuals with different atherosclerotic profiles. Inouye et al. (2018) proposed that patients with increased genetic risk could obtain the maximum benefit from therapies with early intervention in hyperlipidemia and hypertension, generating a subpopulation that benefits from cost-effective primary prevention.

Except for diabetics, there is insufficient research about the association between combined genetic variants and the incidence of major cardiovascular events in patients with known CAD (Sousa et al., 2011; Zhao et al., 2017). The association between these 18 loci and MACE has been established. It is unknown which of these loci has a more significant contribution to coronary events in existing CAD. Variants leading to acute coronary events may diverge from those influencing recurrent MACE since they predispose to plaque instability rather than its formation.

Of the studied 18 genetic variants presenting an $H R>1$, the gene that came closest to statistical significance $(p=0.05)$ was TCF21 rs12190287 and should be further tested in other works as a potential predictor for adverse events (Nurnberg et al., 2015).

Genetic scores could allow for precision approaches in medicine, categorizing patient subgroups at increased risk of recurrent events or those with specific pathophysiology in which a particular targeted therapy or preventive strategy would be more beneficial. Current studies strongly argue that $\mathrm{CAD}$ risk is proportionate to the duration and strength of exposure to hyperlipidemia. Navar-Bogan et al. (2015), based on the Framingham Offspring Cohort, show a two-times increase in CAD risk and cardiac events for each decade of hyperlipidemia exposure (Navar-Bogan et al., 2015; Roberts et al., 2020). Over the subsequent years, the advances in human genomics and proteomics may well allow for identifying protein biomarkers that predict cardiovascular outcomes, including biomarkers involved in regulating homeostasis and inflammatory pathways. Using a targeted proteomics platform, we can extend previous and established novel biomarker associations with the incidence of CVD events, validating past genetic associations (Ho et al., 2018). When genetic sequencing becomes more affordable, as expected, new data can guide efforts in the development of safer, more effective drugs and in helping providers to prevent and treat CVD.

\section{Strengths and limitations}

This study's main strength is the homogeneity of the population, all born and living in a genetically isolated area: Madeira Archipelago. It is a Southern European Caucasian population with no genetic admixtures. Most studies with GRS included thousands of individuals and samples from different cohorts with heterogeneous people, which can be criticized because this heterogeneity can impact the genetic study's results.

Another strength is that this study was explicitly designed to detention a representative population of coronary patients, who had been carefully characterized at baseline, and the predictors have been selected using both clinical and statistical awareness. 
Thirdly, our study had an extended prospective followup, allowing us to study the influence of MACE risk variants on long-term outcomes. It is recognized how difficult it can be to collect and record detailed information about such a large number of patients during an extended follow-up.

Our results must also be taken in the light of possible limitations. Our GRS comprised a group of SNPs whose pathophysiological mechanism may be associated with a clinically relevant increase in the risk of MACE. However, if it included many SNPs associated with different pathophysiological axes, it could be more informative. Future research in genomics, proteomics and gene expression may reveal new markers associated with inflammation, endothelium, thrombosis and new environmental triggers, which help to unravel the complexity of CAD and establish the bases of precision medicine in this field (Eagle et al., 2010; Brunicardi et al., 2011; Leopold and Loscalzo, 2018).

Another major limitation of our study is the sample size. Our findings need to be validated in a larger sample of patients with chronic CAD.

In conclusion: Our wGRS improved CVD risk discrimination and reclassification over and above the conventional risk factors. Besides, we demonstrate that wGRS is more useful in early life, in addition to traditional and clinical risk factors. Further GRS screening could help specifically patients at intermediate risk for cardiovascular events to prevent future episodes by intensive statin and antihypertensive treatments.

\section{Acknowledgements}

The authors are incredibly grateful to the Executive Board of Hospital Central do Funchal for funding this publication. We are thankful to Mrs Elsa Segura for all the administrative support and to Mrs Graça Correia and Mrs Paula Nascimento from the Hospital's library for efficient scientific research. We express our gratitude toward all patients, colleagues, and all collaborators who participated in this study.

This study was supported by the European Regional Development Fund Operational Programmer for the Enhancement of Economic Potential and Territorial Cohesion for the Autonomous Region of Madeira (INTERVIR+).

\section{Conflict of Interest}

The authors declare that no conflict of interest could be perceived as prejudicial to the impartiality of the reported research.

\section{Authors Contributions}

MIM designed and directed the study, wrote the manuscript; EH and SF performed the statistical analysis; ACS, AP, MS, MT, JM, JS and RR performed the clinical assessment and contributed to the database; SB proofread the manuscript technically; GG performed with the DNA extraction and SNPs genotyping; RPR was involved in planning and supervision of the study, discussed the results and commented on the manuscript. All authors read and approved the final version.

\section{References}

American Diabetes Association (2020) Glycemic targets: Standards of medical care in diabetes. Diabetes Care 43:S66-S76.

Assimes TL and Roberts R (2016) Genetics: Implications for prevention and management of coronary artery disease. J Am Coll Cardiol 68:2797-2818.

Backgr Störk S, Feelders RA, van den Beld AW, Steyerberg EW, Savelkoul HF, Lamberts SW, Grobbee DE and Bots ML (2006) Prediction of mortality risk in the elderly. Am J Med 119:519-525.

Benson MD, Yang Q, Ngo D, Zhu Y, Shen D, Farrell LA, Sinha S, Keyes MJ, Vasan RS, Larson MG et al. (2018) The Genetic architecture of the cardiovascular risk proteome. Circulation 137:1158-1172.

Braunwald E and Morrow DA (2013) Unstable Angina: Is it time for a requiem? Circulation 127:2452-2457.

Brehm A, Pereira L, Kivisild T and Amorim A (2003) Mitochondrial 00161 portraits of the Madeira and Açores archipelagos witness different genetic pools of its settlers. Hum Genet 114:77-86.

Brunicardi FC, Gibbs RA, Wheeler DA, Nemunaitis J, Fisher W, Goss J and Chen C (2011) Overview of the development of personalized genomic medicine and surgery. World J Surg 35:1693-1699

Catapano AL, Graham I, De Backer G, Wiklund O, Chapman MJ, Drexel H, Hoes AW, Jennings CS, Landmesser U, Pedersen TR et al. (2016) $2016 \mathrm{ESC} / \mathrm{EAS}$ guidelines for the management of dyslipidaemias. Eur Heart J 37:2999-3058.

Chan YK, Chen Y-F, Pham T, Chang W and Hsieh M-Y (2018) Artificial intelligence in medical applications. J Healthc Eng 2018:4827875.

Cockcroft DW and Gault MH (1976) Prediction of creatinine clearance from serum creatinine. Nephron 16:31-41.

DeLong ER, DeLong DM and Clarke-Pearson DL (1988) Comparing the areas under two or more correlated receiver operating characteristic curves: a nonparametric approach. Biometrics 44:837-845

Demler OV, Pencina MJ and D'Agostino RB Sr (2012) Misuse of DeLong test to compare AUCs for nested models. Stat Med 31:2577-2587.

Dogan MV, Grumbach IM, Michaelson JJ and Philibert RA (2018) Integrated genetic and epigenetic prediction of coronary heart disease in the Framingham Heart Study. PLoS One 13:e0190549.

Eagle KA, Ginsburg GS, Musunuru K, Aird WC, Balaban RS, Bennett SK, Blumenthal RS, Coughlin SR, Davidson KW, Frohlich ED et al. (2010) Identifying patients at high risk of a cardiovascular event in the near future: Current status and future directions - Report of a National Heart, Lung, and Blood Institute. Circulation 121:14471454.

Foley TA, Mankad SV, Anavekar NS, Bonnichsen CR, Morris MF, Miller TD and Araoz PA (2012) Measuring left ventricular ejection fraction - techniques and potential pitfalls. Eur Cardiol 8:108-14.

Futterman LG and Lemberg L (1998) Fifty per cent of patients with coronary artery disease do not have any of the conventional risk factors. Am J Crit Care 7:240-244.

Giampaoli S, Palmieri L, Mattiello A and Panico S (2005) Definition of high-risk individuals to optimise strategies for primary prevention of cardiovascular diseases Nutr Metab Cardiovasc Dis 15:79-85.

Gonçalves R, Freitas A, Branco M, Rosa A, Fernandes AT, Zhivotovsky LA, Underhill PA, Kivisild T and Brehm A(2005) Y-chromosome lineages from Portugal, Madeira and Açores record elements of Sephardim and Berber ancestry. Ann Hum Genet 69:443-454. 
Hajar R (2017) Risk factors for coronary artery disease: Historical perspectives. Heart Views 18:109-114.

Hardy J and Singleton A (2009) Genome-wide association studies and human disease. N Engl J Med 360:1759-68.

Hirschhorn JN and Daly MJ (2005) Genome-wide association studies for common diseases and complex traits. Nat Rev Genet 6:95-108.

Ho JE, Lyass A, Courchesne P, Chen G, Liu C, Yin X, Hwang S-J, Massaro JM, Larson MG and Levy D (2018) Protein biomarkers of cardiovascular disease and mortality in the community. $\mathrm{J}$ Am Heart Assoc 7:e008108.

Inouye M, Abraham G, Nelson CP, Wood AM, Sweeting MJ, Dudbridge F, Lai FY, Kaptoge S, Brozynska M, Wang T et al. (2018) Genomic risk prediction of coronary artery disease in 480,000 adults implications for primary prevention. J Am Coll Cardiol 72:1883-1893.

Jiang J, Zheng Q, Han Y, Qiao S, Chen J, Yuan Z, Yu B, Ge L, Jia J, Gong Y et al. (2020) Genetic predisposition to coronary artery disease is predictive of recurrent events: A Chinese prospective cohort study. Hum Mol Genet 29:1044-1053.

Kandaswamy E and Zuo L (2018) Recent advances in treatment of coronary artery disease: Role of science and technology. Int J Mol Sci 19:424.

Khera AV and Kathiresan S (2017) Genetics of coronary artery disease: discovery, biology and clinical translation. Nat Rev Genet 18:331-344.

Kolber MR and Scrimshaw C (2014) Family history of cardiovascular disease. Can Fam Physician 60:1016.

Kim SY, Lohmueller KE, Albrechtsen A, Li Y, Korneliussen T, Tian G, Grarup N, Jiang T, Andersen G, Witte D et al. (2011) Estimation of allele frequency and association mapping using next-generation sequencing data. BMC Bioinformatics 12:231.

Labos C, Martinez SC, Wang RH, Lenzini PA, Pilote L, Bogaty P, Brophy JM, Engert JC, Cresci S and Thanassoulis G (2015) Utility of a genetic risk score to predict recurrent cardiovascular events 1 year after an acute coronary syndrome: A pooled analysis of the RISCA, PRAXY, and TRIUMPH Cohorts. Atherosclerosis 242:261-267.

Leopold JA and Loscalzo J (2018) The emerging role of precision medicine in cardiovascular disease. Circ Res 122:1302-1315.

Mancia G, Rosei EA, Azizi M, Burnier M, Clement DL, Coca A, Simone G, Dominiczak A, Kahan T, Mahfoud F et al. (2018) ESC/ESH Guidelines for the management of arterial hypertension: The task force for the management of arterial hypertension of the European Society of Cardiology (ESC) and the European Society of Hypertension (ESH). Eur Heart J 39:3021-3104.

Marston L, Carpenter JR, Walters KR, Morris RW, Nazareth I, White IR and Petersen I (2014) Smoker, ex-smoker or non-smoker? The validity of routinely recorded smoking status in UK primary care: a cross-sectional study. BMJ Open 4:e004958.

McCarthy J, Parker A, Salem R, Moliterno DJ, Wang Q, Plow EF, Rao S, Shen G, Rogers WJ and Newby LK et al. (2004) Large scale association analysis for identification of genes underlying premature coronary heart disease: cumulative perspective from analysis of 111 candidate genes candidates. J Med Genet 41:334-341.

McPherson R (2014) Genome-Wide association studies of cardiovascular disease in European and Non-European Populations. Curr Genet Med Rep 2:1-12.

Miao B, Hernandez AV, Alberts MJ, Mangiafico N, Roman YM and Coleman CI (2020) Incidence predictors of Major Adverse Cardiovascular Events in patients with established atherosclerotic disease or multiple risk factors. J Am Heart Assoc 9:e014402.
Moorthie S, Villiers CB, Brigden T, Gaynor L, Hall A, Johnson E, Sanderson S and Kroese M (2019) Polygenic scores, risk and cardiovascular disease. PHG Foundation, Cambridge, $81 \mathrm{p}$.

Navar-Boggan AM, Peterson ED, D'Agostino RB, Neely B, Sniderman AD and Pencina MJ (2015) Hyperlipidemia in early adulthood increases long-term risk of coronary heart disease. Circulation 131:451-458.

Nurnberg ST, Cheng K, Raiesdana A, Kundu R, Miller CL, Kim JB, Arora K, Carcamo-Oribe I, Xiong Y, Tellakula N et al. (2015) Coronary artery disease associated transcription factor TCF21 regulates smooth muscle precursor cells that contribute to the fibrous cap. PLoS Genet 11:e1005155.

Patel RS, Sun YV, Hartiala J, Veledar E, Su S, Sher S, Liu YX, Rahman A, Patel R, Rab ST et al. (2012) Association of a genetic risk score with prevalent and incident myocardial infarction in subjects undergoing coronary angiography. Circ Cardiovasc Genet 5:441-449.

Pencina MJ, D'Agostino RB, Pencina KM, Janssens AC and Greenland P (2012) Interpreting the incremental value of markers added to risk prediction models. Am J Epidemiol 176:473-481.

Pereira A, Mendonça MI, Borges S, Freitas S, Henriques E, Rodrigues M, Freitas AI, Sousa AC, Brehm A and Palma dos Reis R (2018a) Genetic risk analysis of coronary artery disease in a population based study in Portugal, using a genetic risk score of 31 variants. Arq Bras Cardiol 111:50-61.

Pereira A, Mendonca MI, Borges S, Sousa AC, Freitas S, Henriques E, Rodrigues M, Freitas AI, Guerra G, Freitas C et al. (2018b) Additional value of a combined genetic risk score to standard cardiovascular stratification. Genet Mol Biol 41:766-774.

Physical Activity Guidelines Advisory Committee Report, 2008. To the Secretary of Health and Human Services. Part A: executive summary (2009) Nutr Rev 67:114-120.

Rehm J, Greenfield TK, Walsh G, Robson L and Single E (1999) Assessment methods for alcohol consumption, prevalence of high-risk drinking and harm: A sensitivity analysis. Int $\mathrm{J}$ Epidemiol 28:219-224.

Roberts R, Campillo A and Schmitt M (2020) Prediction and management of CAD risk based on genetic stratification. Trends Cardiovasc Med 30:328-334.

Said MA, van de Vegte YJ, Zafar MM, van der Ende MY, Raja GK, Verweij N and van der Harst P (2019) Contributions of interactions between lifestyle and genetics on coronary artery disease risk. Curr Cardiol Rep 21:89.

Smit JA, Ware EB, Middha P, Beacher L and Kardia SL (2015) Current applications of genetic risk scores to cardiovascular outcomes and subclinical phenotypes. Curr Epidemiol Rep 2:180-190.

Sousa AG, Lopes NH, Whady AH, Krieger JE and Pereira AC (2011) Genetic variants of diabetes risk and incident cardiovascular events in chronic coronary artery disease. PLoS One 6:e16341.

Spodick DH, Raju P, Bishop RL and Rifkin RD (1992) Operational definition of normal sinus heart rate. Am J Cardiol 69:1245-1246.

Steyerberg EW, Vickers AJ, Cook NR, Gerds T, Gonen M, Obuchowski N, Pencina MJ and Kattan MW (2010) Assessing the performance of prediction models: a framework for some traditional and novel measures. Epidemiology 21:128-138.

Tabor HK, Risch NJ and Myers RM (2002) Candidate gene approaches for studying complex genetic traits: practical considerations. Nat Rev Genet 3:391-397.

Thygesen K, Alpert JS, Jaffe AS, Chaitman BR, Bax JJ, Morrow DA, White HD, Executive Group on behalf of the Joint European Society of Cardiology (ESC)/American College of Cardiology (ACC)/American Heart Association (AHA)/World Heart Federation (WHF)/Task Force for the Universal Definition of Myocardial Infarction. Fourth Universal Definition of Myocardial Infarction (2018) J Am Coll Cardiol 72:2231-2264. 
Watkins H and Farrall M (2006) Genetic susceptibility to coronary artery disease: from promise to progress. Nat Rev Genet 7:163-173.

Wellcome Trust Case Control Consortium (2007) Genome-wide association study of 14,000 cases of seven common diseases and 3,000 shared controls. Nature 447:661-667.

Zhao C, Zhu P, Shen Q and Jin L (2017) Prospective association of a genetic risk score with major adverse cardiovascular events in patients with coronary artery disease. Medicine 96:51.

\section{Supplementary Material}

Table S1 - Genetic variants associated with CAD risk in the GENEMACOR population $(\mathrm{n}=3120)$.

Associate Editor: Mara Hutz

License information: This is an open-access article distributed under the terms of the Creative Commons Attribution License (type CC-BY), which permits unrestricted use, distribution and reproduction in any medium, provided the original article is properly cited. 